\title{
Special Issue Dedicated to the 13th International Symposium on Parameterized and Exact Computation
}

\section{${\text { Christophe } \text { Paul }^{1} \cdot \text { Michał Pilipczuk }}^{2}$}

Published online: 25 July 2020

๑) Springer Science+Business Media, LLC, part of Springer Nature 2020

We are pleased to present this special issue of Algorithmica to the 13th International Symposium on Parameterized and Exact Computation (IPEC) held in Helsinki, Finland. IPEC (formerly IWPEC) is a series of international symposia covering research in all aspects of parameterized and exact algorithms and complexity. Started in 2004 as a biennial workshop, it became an annual event in 2009 and is now a highly recognized annual meeting. This special issue is a successor of following special issue volumes of Algorithmica: volumes 64(1)-2012 and 65(4)-2013 dedicated to IPEC 2010, held in Chennai (India); volume 71(3)-2015 dedicated to IPEC 2013, held in Sophia Antipolis (France); volume 75(2)-2016 dedicated to IPEC 2014, held in Wrocław (Poland); volume 79(1)-2017 dedicated to IPEC 2015, held in Patras (Greece); volume 81(2)-2019 dedicated to IPEC 2016, held in Aarhus (Denmark); volume 81(10)-2019 dedicated to IPEC 2017, held in Vienna (Austria).

The current volume comprises a selection ten extended journal papers that were presented at IPEC 2018. Among the twenty-eight papers presented at IPEC, twelve were originally invited to submit their full version to this volume. After a rigorous reviewing processing, according to the high standard of the journal, ten of them were accepted for publication.

In their paper entitled "Best-case and Worst-case Sparsifiability of Boolean CSPs", H. Chen, B. M. P. Jansen and A. Pieterse provide algorithmic results in the context of polynomial-time sparsification for NP-complete Boolean Constraint Satisfaction Problems.

The paper entitled "Multi-budgeted directed cuts", by S. Kratsch, S. Li, D. Marx, Ma. Pilipczuk and M. Wahlström, studies enriched version of important parameterized cut problems, namely Skew Multicut and Directed Feedback Arc Set. The authors

Christophe Paul

christophe.paul@lirmm.fr

Michał Pilipczuk

michal.pilipczuk@mimuw.edu.pl

1 LIRMM, Montpellier University, CNRS, Montpellier, France

2 MIMUW, University of Warsaw, Warsaw, Poland 
design parameterized branching algorithms using an extension of the important separator technique.

In "Dual parameterization of Weighted Coloring", J. Araújo, V. Campos, C. V. Lima, V. F. dos Santos, I. Sau and A. Silva explore the parameterized tractability of coloring problems, known to be hard with respect to some parameter $k$, when parameterized by $n-k$, where $n$ is the weighted input size. The results include FPT algorithms, polynomial kernels and kernelization hardness proofs.

The paper "Multivariate Analysis of Orthogonal Range Searching and Graph Distances" by K. Bringmann, T. Husfeld and M. Magnusson presents a parameterized complexity analysis of basic problems belonging to $\mathrm{P}$ such as computing the diameter, the radius or the Wiener index of a graph. Treewidth and vertex cover are the parameters considered in this research.

In "Parameterized Complexity of Independent Set in H-Free Graphs", É. Bonnet, N. Bousquet, P. Charbit, S. Thomassé and R. Watrigant answer an open question on the Maximum Independent Set problem in $C_{4}$-free graphs. They provide hardness proofs as well as algorithmic results, including Turing kernels.

The paper "Counting Induced Subgraphs: A Topological Approach to \#W[1]-hardness", by M. Roth and J. Schmitt, investigates the problem of counting in an input graphs the number of subgraphs of size $k$ satisfying a given property. The authors establish hardness results for various properties, including (dis-)connectedness and the parity of the number of edges.

In "Parameterized Leaf Power Recognition via Embedding into Graph Products", D. Eppstein and E. Havvaei propose new advances towards the resolution of a longstanding open problem, namely the $k$-leaf power recognition problem (for $k \geq 7$ ). Their approach relies on embedding the input instance in a graph product of bounded treewdith, and then using monadic second order logic or dynamic programming.

In the paper "A Faster Tree-Decomposition Based Algorithm for Counting Linear Extensions", K. Kangas, M. Koivisto and S. Salonen propose a dynamic programming algorithm involving fast multiplication of multivariate polynomials in order to count the number of linear extension of a poset whose cover graph has bounded treewidth.

The paper "On the Distance Identifying Set meta-problem and applications to the complexity of identifying problems on graphs" by F. Barbero, L. Isenmann and J. Thiebaut investigates the algorithmic and complexity aspects of a new parameterized problem which is a common generalization of several important problems such as Identifying Code, Locating Dominating Set and Metric Dimension.

In the paper entitled "The parameterised complexity of computing the maximum modularity of a graph", K. Meeks and F. Skerman explore the parameterized tractability of the maximum modularity problem under standard structural parameters such as vertex cover, treewidth and pathwidth, maximum leaf number.

The editors would like to thank all authors and reviewers for contributing to this special issue. We would also like to express our appreciation to the editorial staff of Algorithmica for their assistance.

Publisher's Note Springer Nature remains neutral with regard to jurisdictional claims in published maps and institutional affiliations. 